\title{
Scope and Reliability of Modern to Ancient System of Medicine: A Need to Develop Best Practices, Formulations and Scaling for Better Health Care
}

\author{
Ravi Kant Upadhyay* \\ Department of Zoology, DDU Gorakhpur University, India
}

Submission: April 11, 2017; Published: May 30, 2017

"Corresponding author: Ravi Kant Upadhyay, Department of Zoology, DDU Gorakhpur University, India, Email: rkupadhya@yahoo.com

\begin{abstract}
Present article emphasizes scope and reliability of modern to ancient system of medicine. For better therapeutic success there is a need to integrate all existing systems of medicine under one umbrella to make better formulation for beating drug resistant microbes mainly diseases and infections. To replace outdated drugs both resistant, less effective and old system medicine there is a need to develop plant based herbal medicines for daily usage because chemical drugs are toxic in nature and impose long term cumulative side effects. Due to high costs chemical drugs are not easily affordable for millions of poor round the globe. Today human life is full of stress due to changes in behavioral environment and there is a rising trend of life style and communicable diseases with an alarming rate of inborn and neonatal morbidities related to obesity, diabetes, cancer and cardiovascular disorders. There is a crying need of new more effective medicines in developing and under developed countries which could have low dosage, low cost and possess lesser side effects in comparison to synthetic drugs with high target specificity. There was felt a need of development of a more comprehensive medical system that can provide preventive, curative and rehabilitative healthcare to masses. It will also need fast and accurate diagnosis and time bound treatment modalities based on scientific principles with one holistic approach and fusion of various medicinal systems prevalent. For increasing better coverage and disease curing efficacy and reduce the serious adverse effects of toxic and microbial resistant drugs. There should be an umbrella of safe and non toxic medicines that can provide low cost drug formulae for larger therapeutic cover to the large section of the people. It will not only provide them physical well being but also tone up health of body, mind and soul. There is a need to carry ethnobotany, pharmacognosy, pharmacology, pharmaceutics to link allopathic, homeopathic, Ayurvedic, cultural, social and traditional medicines on single platform to adjudge there capability to revitalize the therapeutic potential of thousands of drug regimens. There are series of biomaterials which are new to the market, still unidentifiable or remain unscreened till date but may be of high therapeutic use. It will certainly give new insights in developing potentially new bio-actives for formulation of quality life saving drugs mainly of plant origin after having proper formulation, testing, processing, scaling and marketing.

Keywords: Plant biomaterial; Herbal medicines; Ethnobotany; Pharmacognosy; Pharmacology; Pharmaceutics; Communicable diseases; Life style diseases
\end{abstract}

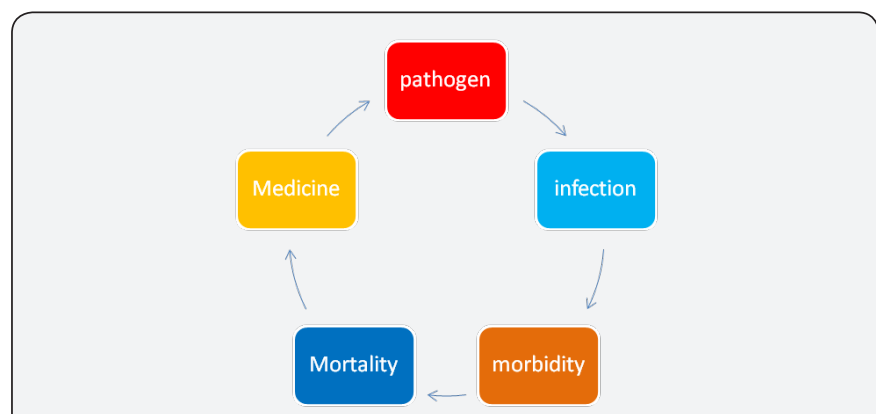

Figure 1: Showing various cycle of disease occurrence and need of medicine.

In modern age there is a rising problem of life style and communicable diseases with an alarming rate of inborn and neonatal morbidities related to obesity, diabetes, cancer and cardiovascular disorders. Naturally, disease pathogens found in different climatic areas with different environmental adaptation but adopt nearly similar annual cycle of disease occurrence and its prevalence (Figure 1). There are so many plant families which have series of bio-organic compounds which were synthesized in anticipation to pathogens and pest population. Meanwhile important aspect is to screen out all broad spectrum antipathogenic and disease curing biomaterials. Round the world old civilizations and ethnic groups have plethora of experience based traditional medicines, which are slow acting but non toxic and are of low cost. Very simply particular substrate needs catabolism to release out energy filled in form of chemical bonds and also need a series of suitable catabolic enzymes or 
an antibody is synthesized in response to an antigen, its shows that nature is very sensibly work to synthesize deterrents, repellents, attractants wherever if feels a need. Conceptually drugs are not synthesized until and unless need is not felt and placed accordingly. Second important thing is that whole of therapeutics/pharmaceutics has been already done by nature with the time during long evolution.

Though plant based biomaterials are easy to procure but their drug formation and composition is too tough, because there is huge depository on plant materials is available that has thousand of compounds from various groups of plants. Due to inadequate knowledge of their mode of action, potential adverse reactions, contraindications, and interactions it is very difficult to decide therapeutic use and dose. There are existing orthodox pharmaceuticals and functional foods which need long time promotion after proper testing for both safe and rational use of these agents [1]. Since safety continues to be a major issue with the use of herbal remedies, it becomes imperative, therefore, that relevant regulatory authorities put in place appropriate measures to protect public health by ensuring that all herbal medicines are safe and of suitable quality. It is also common knowledge that the safety of most herbal products is further compromised by lack of suitable quality controls, inadequate labeling, and the absence of appropriate patient information [2]. Plant based medicines are best medicines because of their low toxicity, lesser side effects and easy to catabolize.

Modern pharmaceutical industries can use old formulas and test them to prepare more promising low cost medicines to provide therapeutic cover to low income consumers effectively round the globe. A positive market of modern medicine can be developed by screening their effectiveness, usage, and safe drug assimilation. It will need to clarify and find solutions of true critics from the users and their regular improvement to have many more modern medicines which may be well tested and properly designed with sustainable branding to make attractive in the eyes of consumers round the globe, Acceptability of drug should be based on peoples therapeutic index and usage not on the basis of commissions collected from the market. It is the time when all life saving medicines should become license free and low cost. There is a need to increase the social acceptability of modern drugs from all systems of medicine as a part of a global counter culture marked by utmost human needs, sociality, ethnicity and spirituality.

Despite treatments available by different medicinal systems the success rate of drug therapeutics is not very high for chronic diseases. In present time lot of drug formulae are floating in the market, but they are not providing desirable effectiveness and cure. Even today African and Asian countries are facing very high infection rate and prevalence of communicable diseases due to rising population of pesticide resistant insect vectors. Similarly, microbial infections are also making diverse morbidities and causing high mortality due to presence of drug resistant strains. There is a sharp increase in case of drug resistant $\mathrm{M}$. tuberculosis (MDR) strain infected patients. South East Asia is having many endemic protozoans, bacterial and viral diseases and soil nematodes and parasites as disease causing agents. Both gastric and pulmonary infections are rising alarmingly. Hence, for achieving greater tackling emerging global threats evidence-based policy is needed that can address all risk factors of chronic diseases. Though modern medicinal system is upgraded and providing diagnosis, treatment and care to the people, but it will need multidisciplinary approach to enhance application of science, technology, engineering (mathematics) for the delivery of care. To revitalize the modern medicine system and seeking high therapeutic coverage every country will need a comprehensive, long-term master plan to replace costly drug regimens which are beyond the limit of purchasers/consumers round the globe. There must be cut-down of interstate taxes, announcing subsidies on manufacturing and costs. Both should cover under essential all time health insurance plan. It will also need an integrated and coordinated stewardship in all parts of the health system should be considered to implement reforms efficiently.

\section{Fusion of Traditional Medicines}

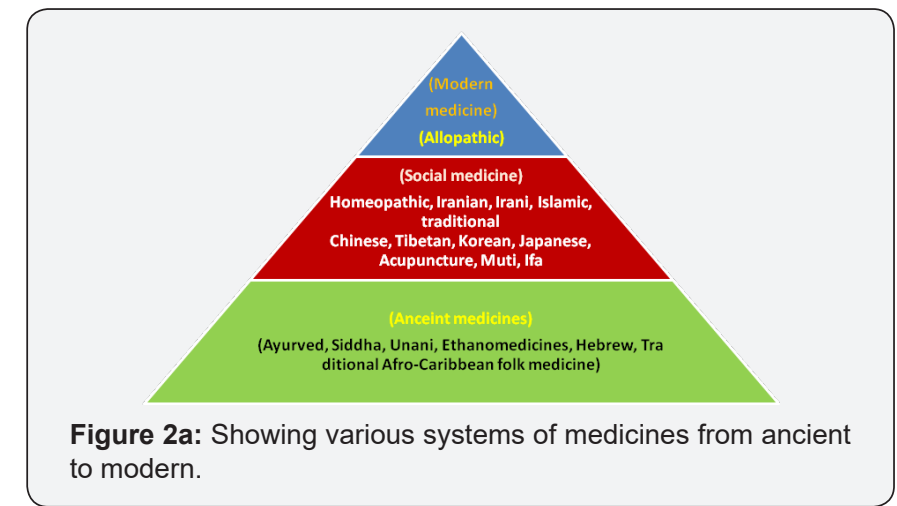

Human being has explored many medicines in the past by direct observations collected from animals by their direct use. Traditional medicines are pre-scientific forms of medicine also known as indigenous or folk medicine comprises knowledge systems that developed over generations within various societies before the era of modern medicine. The WHO defines traditional medicine as "the sum total of the knowledge, skills, and practices based on the theories, beliefs, and experiences indigenous to different cultures, whether explicable or not, used in the maintenance of health as well as in the prevention, diagnosis, improvement or treatment of physical and mental illness. Various traditional medicines were practiced for centuries by human by making timely changes in drug composition, dosage and application. In some Asian and African and countries, up to $80 \%$ of the population relies on traditional medicine for their primary health care needs. When adopted outside of its traditional culture, traditional medicine is often called alternative medicine. There are thousands of ethno-medicines mainly tribal formulation which were found highly effective than allopathic 
medicines and are of low cost [3]. Practices known as traditional medicines include Ayurveda, Siddha, Unanim anceient Iranian, Irani, Islamic, traditional Chinese, Tibetan, Korean, Japanese, acupuncture, Muti, Ifa and ancient Hebrew medicine traditional Afro-Caribbean folk medicine [4] (Figure 2a \& 2b).

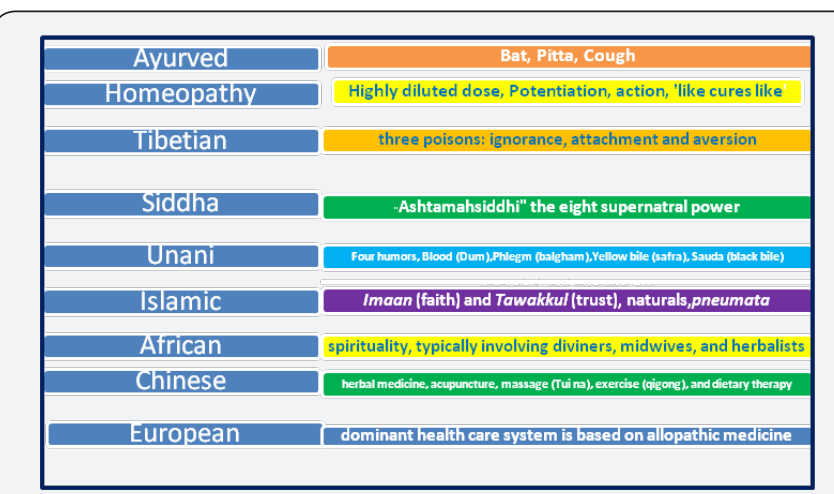

Figure $\mathbf{2 b}$ : Showing principles of various systems of medicines from ancient to modern.

According to WHO policy on health there should be universal health coverage (UHC) that will cover people and communities from the entire world and use the promotive, preventive, curative, rehabilitative and palliative health services that they need. It should be of sufficient quality, more effective and ensuring use of these services to and does not expose the user to financial hardship. Traditional medicine may include formalized aspects of folk medicine, that is to say longstanding remedies passed on and practiced by lay people. Folk medicine consists of the healing practice practices and ideas of body physiology and health preservation known to some in a culture, transmitted informally as general knowledge. There is strong demerit they have no evaluative literature for their bioefficacy and are practiced by layman or by anyone in the culture having prior experience. Folk medicine may also be referred to as traditional medicine, alternative medicine, indigenous medicine or natural medicine [3]. In recent years modern science has validated number of teachings and beliefs rooted in the ancient wisdom of Unani Science. This has vast literature, evidences and is a comprehensive medical system that provides promotion, prevention, fast cure and rehabilitative healthcare. Its fundamentals, diagnosis and treatment modalities are based on scientific principles and a holistic approach considering each individual in relation to his/her environment and emphasizes on the health of body, mind and soul. Unani system of medicine based on humoural theory and postulates the presence of four humours in the body. These are dam (blood), balgham (phlegm), safra (yellow bile) and sauda (black bile), a parallel to kapha, vata and pitta, the three doshas in Ayurveda. The Tibetan medical system is one of the world's oldest known medical traditions. Traditional Tibetan medicine is also known as SowaRigpa medicine, is a centuries-old traditional medical system that employs a complex approach to diagnosis, incorporating techniques such as pulse analysis (Nari) and urinalysis (Urine), and utilizes behavior (Mood) and dietary (Diet). It is an integral part of Tibetan culture and has been developed through many centuries. Tibetan medicine follows Buddha's four noble truths (Dukkha, Samudaya, Nirodha, Magga) which apply medical diagnostic logic to suffering (Figure $2 \mathrm{a} \& 2 \mathrm{~b}$ ). But it is common in all medicinal system that they based on plant materials either raw or processed $[5,6]$. There is a large conflict between allopathy and traditional medicine about preparation, usage, effectiveness and dose related effects. Hence, there is a need to solve preventive-curative conflict in primary health care [7]. It can only solved through proper analytical authentication, and evaluation of medicine by using randomized controlled trials

Thousands of bioactive preparations are available from various medicinal systems such as Ayurvedic Chinese, Tibetian, Unani (Greco-Arab or Unani Tibb), homeopathic and ethnic medicines which can be proved best-selling medicine if screened, modified and supported properly. In Ayurveda, already Caraka samhinta a largest Compendium (c. 200 CE) has thousands of medicinal compositions, applications, with well defined dosage. Cyavanaprāsh is a patriotic formula that boosts body immunity, a modern geriatric drug, and one of the elements in canonical Ayurvedic treatments. Most commonly Ayurveda represents a unique way of looking upon health, disease and the human body [8]. Hence, there is a need to carry ethnobotany, pharmacognosy, pharmacology, pharmaceutics to link allopathic, homeopathic, Ayurvedic, cultural, social and traditional medicines on single platform to adjudge there capability to revitalize the therapeutic potential. There is a need to adopt national policy on traditional medicine and regulation of herbal medicines [9] for practicing of less expensive medicines, highly effective medicines [10]. It will also need proliferation of important brands in the market. Efficient spending on health can promote growth (Figure 2a \& 2b).

Homeopathy is the system of medicine which works on the principle of 'like cures like' (Similia Similibus curanter). This system of holistic healing gives a highly diluted dose, so as to prevent side effects. This minute dose, creates a similar disorder in the vital force and provokes the vital force to react to the symptoms and overcome. The system works similar to a vaccine, which work against any pathogen e.g. small-pox is done by injecting the small-pox disease-causing organism, so that the body can build up its natural immunity. This is called as 'potentisation' of drug chronic diseases. Homeopathy is the best way to cure both acute e.g. fever, cough, cold to asthma, skin diseases and chronic diseases like cancer CVD and diabetes. The theory behind the working of homeopathy is that the body of every human being contains a vital force within the body which regulates the functioning of the body. Due to reasons such as heredity, environmental conditions, stress etc, this vital force weakens causing disease. So, disease is nothing but a complex of certain symptoms observed in the human body. The homeopathic physician makes a study of not only the symptoms the patient is complaining of, but of the entire patient himself. This leads to a picture of the patient. The physician then 
prescribes homeopathic medicine, which matches this picture. Homeopathy uses an array of medicines whose source ranges from animals to plants and chemicals. Plants like Onion (Allium), Capsicum, Belladonna, Cactus, etc are used for preparation of homeopathic medicines. A large number of chemicals like nitric acid, arsenious acid and iron, phosphorous are popularly used in homeopathic remedies (Figure 2a \& 2b).

All these are not only just curable but the cure is mild (without side effects) and permanent. While allopathy makes use of strong medicines to cure, which cause severe side effects, homeopathic medicines are not only sweet and east to take, they work highly effectively without any side effect. The danger of allopathic medicines is that they do not cure but suppress. Cases of acute diseases/disorders like headache, fever, cold, cough, etc can be cured within minutes through the proper selection of the homeopathic remedy. However chronic diseases like asthma, heart problems, cancer etc need time to cure.

\section{Current Status of Diseases and Fatalities}

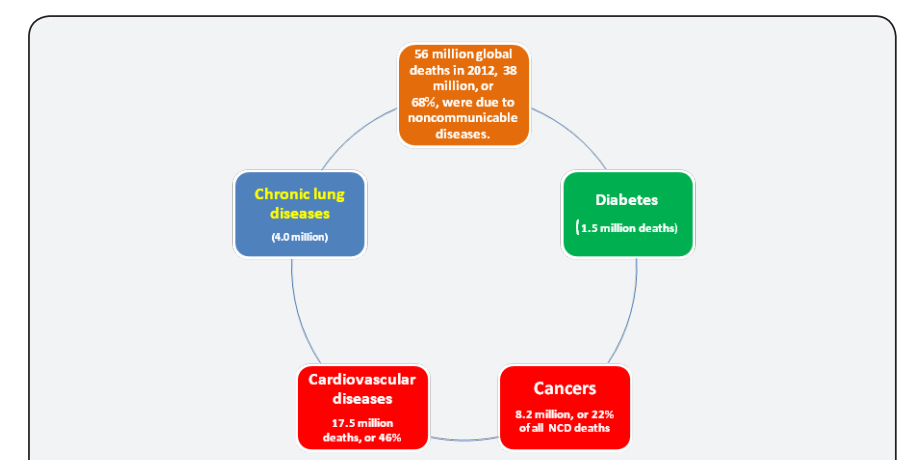

Figure 3: Showing NCD mortality and morbidity at global level occurred in 2012.

According to Global status report on non-communicable diseases 2010, published by World Health Organization; noncommunicable diseases (NCDs) are the biggest global killers today. Sixty-three percent of all deaths occurred in year 2008 (36 million people) were caused by NCDs [11] (Figure 3). Nearly $80 \%$ of these deaths occurred in low- and middle-income countries, where the highest proportion of deaths under the age of 70. In future, there is larger possibility that prevalence of NCDs will increase in low- and middle-income countries and that may cause large number of deaths, particularly, due to population growth and ageing, in conjunction with economic transition and resulting changes in behavioral, occupational and environmental risk factors [12]. By 2020 the largest increase in NCDs will occur in Africa and other low and middle-income countries with large number of deaths. Obesity is now a worldwide epidemic disease and poses a major risk for diet related diseases like type 2 diabetes, cardiovascular disease, stroke and fatty liver among others disorders like diabetes, cancer, hepatitis and hypertension, blood pressure.

NCDs are the most frequent causes of death in most countries in the Americas, the Eastern Mediterranean, Europe, South-East
Asia, and the Western Pacific [11]. In the African Region, there are still more deaths from infectious diseases than NCDs. Even there, however, the prevalence of NCDs is rising rapidly and is projected to cause almost three-quarters as many deaths as communicable, maternal, prenatal, and nutritional diseases by 2020 , and to exceed them as the most common causes of death by 2030 . NCD deaths are projected to increase by $15 \%$ globally between 2010 and 2020 (to 44 million deaths). The greatest increases will be in the WHO regions of Africa, South-East Asia and the Eastern Mediterranean, where they will increase by over 20\% (Figure 3). In contrast, in the European Region, WHO estimates there will be no increase? In the African Region, NCDs will cause around 3.9 million deaths by 2020 . The overall pressure and disease burden will increase almost two fold by 2020 in Asian and African countries and people will face mortality, morbidity with risk factors [11]. The condition will substantially increased because of tobacco, alcohols, use of drugs, insufficient physical activity, raised blood pressure, overweight and obesity, raised cholesterol and cancer-associated infections related with life style of people. Easy treatment are available for diarrhea, hemorrhoids, osteoporosis, diabetes, eye and ear infections, malaria, filarial, skin diseases and gynecological disorders but for chronic diseases like HIV-AIDS and cancer will be of no use.

With advent of modern methods of therapeutics and high through put and fast diagnostic tools and instruments, there is a need to properly test formulations from different medicinal system for their dose determination and responses at cellular and molecular level. For treatment and cure diseases low cost medications and healing methods are to be used. But for better treatment economy is one of the important factors. Even toady people with poor economies are vulnerable and facing adversities of environment, communicable and non communicable diseases not annual basis but full life time. These people get sicker sooner and die earlier than their counterparts in wealthier societies. In large parts of the developing world, non-communicable diseases are detected late, when patients need extensive and expensive hospital care for severe complications or acute events. Poor countries hardly have diagnosis facilities, no vaccination, and almost no hospital care. Most of hospital care is covered through out-of-pocket payments, leading to catastrophic medical expenditures. Due to occurrence of communicable diseases annually and prevalence of non-communicable diseases there are seen heavy losses of national income. Disease burden and hospital care push millions of people below the poverty line, each and every year. It could only manage by rich societies with strong will power, good medical research, and development capacities. Effective interventions are available, and abundant evidence now demonstrates their clear and measurable impact in a range of resource settings. Not only hospitals and research centers could ensure well being of the people societal, demographic and psychological changes are needed to solve the problem.

All essential parameters related to demographic structure, planning, housing, sanitation, waste disposal, vector control, 
community health centers, trainings, field surveys, surveillance and monitoring, mass vaccination and larger drug coverage, comprehensive approach will change the global health related issues and enhancement of lifestyles. The United Nations General Assembly passed a resolution on the prevention and control of non-communicable diseases adopted in 2010. The main obstacle in treatment is economy, the main reason of disease occurrence in low income countries is economy, and they don't have money to purchase drugs, hospitals, nursing and care centers. In one part of the world people are having every treatment and quality health maintenance system. This is the main reason that high-income countries shows that a comprehensive focus on prevention and improved treatment following cardiovascular events has led to dramatic declines in mortality rates. Similarly, scenario is in cancer treatment as an early detection and screening interventions have improved survival rates for many cancers in high-income countries. Survival rates in low and middle-income countries, is very low $[11,12]$.

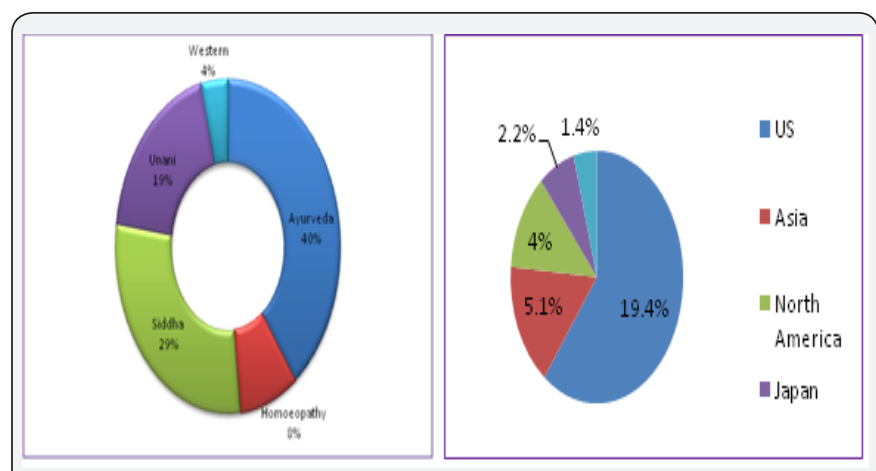

Figure 4a: Showing Indian market of different categories of medicines 2016, 4b worldwide sale of herbal medicines 2002 .

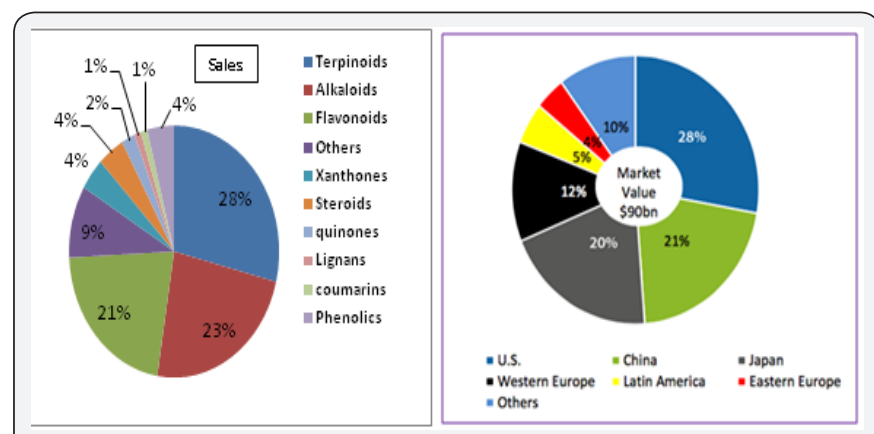

Figure 4c: showing worldwide sale of plant bioorganic compounds 2017, Global vitamin and supplement market share (\%), 2013.

In high income countries cost-effective interventions are available with broad approaches to cancer prevention and control. These are primary prevention, early detection, treatment and palliative care. Early diagnosis based on awareness of early signs and symptoms improves survival, particularly for breast, cervical, colorectal, skin and oral cancers. Almost similar condition is in case of chronic respiratory diseases. In many low-income countries, drugs for inhalation use, such as inhaled steroids, are still not financially accessible. Countries could explore procurement of quality assured inhaled drugs at affordable costs. Lung health programs developed to address tuberculosis might be integrated with interventions for chronic respiratory diseases. While advice and recommendations are universally relevant, the report gives particular attention to conditions in low- and middle-income countries, which now bear nearly $80 \%$ of the burden from diseases like cardiovascular disease, diabetes, cancer and chronic respiratory diseases [11,12] (Figure 4a \& 4d).

There must be clear policy that prevention of any disease should included as a priority in national development initiatives and related investment decisions Depending on the national situation, strengthening the prevention and control of NCDs should also be considered an integral part of poverty reduction and other development assistance programs. All methods, medicines, therapies, hospital care, and treatments should be associated with sustainable development. In this process civil society institutions and groups will influence political and public awareness and make control efforts that will play positive role in control of not only simple diseases but also epidemics. Among major causes of growing burden of NCD diseases are globalization, rapid unplanned urbanization and increasingly sedentary lives. People in developing countries are increasingly eating foods with higher levels of total energy and are being targeted by marketing for tobacco, alcohol and junk food, while availability of these products increases. Overwhelmed by the speed of growth, many governments are not keeping pace with ever-expanding needs for policies; legislation, services and infrastructure that could help protect their citizens from NCDs $[11,12]$. Similarly in case of communicable diseases presence of sanitation, large vector population, precipitation, hosts, lower protection, humidity, temperature and annual rain cycle, pesticide and drug resistance, and no vaccination are important reasons.

\section{Rising Problem of Drug Resistance}

One of the important demerits of modern medicines or synthetic medicines is that most of the microbes have developed resistance against them. Microbes have reduced their effectiveness by cleaving drug structures by enzymatic seizures, and most of the drugs become failed to antimicrobial, anthelmintic or an anti-neoplastic or disease curing potential. There are four different mechanisms by which microbes acquire resistance to antimicrobials i.e. drug inactivation or modification, alteration of target site, and metabolic pathway and reduced drug accumulation. Bacteria are capable of not only altering the enzyme targeted by antibiotics and also the use of enzymes to modify the antibiotic itself and neutralize it. These best examples are Staphylococcus aureus [13], vancomycinresistant enetrococci. Pseudomonas aeruginosa is an antibioticmodifying microbe while Acinetobacter baumannii is an aminoglycoside-resistant microbe. Drug resistance in malaria 
parasite Plasmodium falciparum is increasing day by day against conventional antibiotics such as artemisinin $[14,15]$. Emergence of multiple drug resistance in P. falciparum, leads to global resurgence of malaria in the last three decades. Against malaria parasite almost old prescriptions are totally failed $[15,16]$ and it became quite disastrous [17]. Viruses, fungi and parasites still exist which are on path of rising multidrug resistance. Pan drug resistance also exists.

In last three decades HIV virus has also acquired drug resistance [18]. There is a complete treatment failure, because medicines can't prevent the drug-resistant HIV from multiplying. Similarly, drug resistant TB is also increasing day by day. Due to higher prescription of antimicrobials TB bacteria acquired drug resistance and its treatment is inadequate. There are several reasons that wrong TB drugs or sub standard were prescribed to the patients that resulted drugs resistance. Secondly, transmitted or primary drug resistant $\mathrm{TB}$, results from the direct transmission of drug resistant TB from one person to another. The occurrence and prevention of primary drug resistant TB has largely been neglected during the development of global programs to end TB. Multi drug resistant TB has resistance against isoniazid and rifampicin treatments. There is another category of TB that is XDR-TB (extensively drug resistant TB) which is resistant to at least rifampicin and isoniazid [19]. This is in addition to strains being resistant to one of the fluoroquinolones, as well as resistant to at least one of the second line injectable TB drugs amikacin, kanamycin or capreomycin.

\section{Plant Natural Products}

The main category of plant natural products is plant essential oils which are secondary metabolites and possess broad spectrum antimicrobial activity. Essential oils isolated from different plant species contain lectins, polypeptides, alkaloids, phenolic compounds, quinines, flavonoids, flavones, flavanoles, terpenes, terpinoids, coumarins are important phytochemicals which show activity against bacteria, fungi, viruses, protozoans, helminthes and insects . These compounds also showed immense anti-microbial, anticancer and anti-tumor potential. All these can be used as a source material to prepare various composite nanomedicines and more popular drug formulations. Other plant natural products such as resins, latex, powders, plant sap, root stem, flowers and fruits are used as source materials for many medicinal formulations. Gum resins isolated from Boswellia species, also known as frankincense, are major ingredient in Ayurvedic and Chinese medicine. Boswellia gum resins essential oil exhibits anti-neoplastic, anti-tumor and anticancer activity against human pancreatic cancer [20]. Aristata extracts and its formulations were found highly useful to treat diarrhoea, haemorrhoids, gynaecological disorders, HIVAIDS, osteoporosis, diabetes, eye and ear infections. It is also used in wound healing, jaundice, skin diseases and malarial fever. There are so many plant species which may be useful as anti- pyretic, anti-bacterial, anti-microbial, anti-hepatotoxic, antihyperglycaemic, anti-cancer, anti-oxidant and anti-lipidemic agent.

Besides pure herbal compounds metallic nanoparticles can be used for the development of a new-generation of nano-devices. There is a possibility natural fusion of nano-metallic compounds can be made inside plant cells in culture. More often, plant based nanoparticles if used as homeopathic medicinal ingredients possibly a new integrated model for many therapeutic treatments may develop. Silica nanostructures formed during successions in glass and/or biosynthesized by specific plant extract tinctures can also use as epitaxial source materials to have higher potencies of homeopathic medicines. Such fusion of nanoremedies if prescribed in low doses will act much faster than conventional drugs over time and give more curative biological signals. In addition, diverse nanoemulsion vehicles (NEs) have been developed with vast potential for improving therapeutic index of clinically approved and experimental drugs. Using oils rich in omega-3 and omega- 6 polyunsaturated fatty acids (PUFA), several promising nano-emulsion formulations have been developed recently for oral and systemic administration. Curcumin a bioactive component isolated from the rhizomes of turmeric Curcuma longa Linn. (Zingiberaceae); a commonly used spice and nutritional supplement. It shows much better potential pharmacological action in inflammatory disorders, cardiovascular disease, cancer, Alzheimer's disease and neurological disorders. Similarly garlic is a good depository of sulfur compounds with multiple biological activities can be used for finding new nano-medicine making candidate molecules. Thymoquinone is the main bioactive compound of Nigella sativa that can be used as an important drug carrier. There are thousands of plants available which are still unscreened that can be used as good anti-pyretic, anti-bacterial, anti-microbial, antihepatotoxic, anti-hyperglycaemic, anti-cancer, anti-oxidant and anti-lipidemic agent. B. aristata extracts and its formulations are also useful in the treatment of diarrhoea, haemorrhoids, gynaecological disorders, HIV-AIDS, osteoporosis, diabetes, eye and ear infections, wound healing, jaundice, skin diseases and malarial fever.

\section{Search for New Biomaterials and Bioinformatics Tools}

After seeing the rising cost of conventional drugs there is a need to have low cost drugs with wider molecular and biopharmaceutical significance. On other side scientific community is involved in searching novel antigens for vaccination, nano-drug structures and scaffolds as medicine, and searching new methods for drug delivery to combat few noncurable dreadful diseases. However, to cut down existing high costs of medicine there is a need to screen plant biomaterials that can be used to prepare low cost drug formulations. For fine processing, and making easy and appropriate drug targets it 
will need integrated research in computational science, atomic structural biology, bioinformatics, virtual drug design, genomics and biological networks. Further, better tools will be required to design new interacting bio-molecules by making slight changes or additions based on homology modeling with multiple molecular dynamics simulation for identifying representative conformation of important human receptor sub types with interacting pathogen antigens. All key biological factors, responsible for the variation in the conservation rate of different disordered regions within the human proteome, proteins should identify. However, protein domains preferably having disordered regions will be explored to enhance their structural flexibility which in turn will facilitate their molecular interactions.

There is need to study in-vitro and in-vivo behaviors and biodistribution of nano-materials by using modern analytical tools such as synchrotron-based X-ray microscopy. Technological upgradation that will unfold complex interactions of drugs and receptors at the nano-biological interface and account unique biological of nanomaterials should be made. Nanoparticles based on plant viruses are emerging biomaterials for medical applications such as drug delivery and imaging will be investigated to explore their regular structures, possible genetic and chemical modifications to carry large payloads of cargos, as well as targeting ligands. Ultramodern tools must be used to explore structure and function of nucleic acids, nucleotides, proteins, peptides, membranes, polysaccharides and all their components, metal complexes and model systems. Raman spectroscopy is still a suitable and prominent method for probing the relationship between structure, dynamics and function of biomolecules. Microarray technologies can solve the problem related to serial gene expression patterns and mutations. Combination of X-ray and neutron crystallography, NMR, Tetrahertz spectroscopy, mass spectroscopy, thermodynamics and computer simulations help to reveal how water assist in their protein function. $\mathrm{z}$ Time-resolved optical, rapid mixing techniques - folding \& function mechanisms. New innovation is needed in biophysics, spectroscopy, lasers and bio-sensing technology to perform in-silico simulation based modular studies and to explore molecular basis of drug action of life saving antibiotics. There is a need to test biomolecules used in various systems of medicine should pass through three-dimensional imaging mainly biomedical imaging and holographic displays for deciding their better role in modern pharmaceutical industry. With advent of new formulations clinicians should also need exhaustive training for fast therapeutic judgment. There is a need to make organizational, psychosocial, biomedical, and societal framework to support health awareness and healthcare.

\section{Development of Drug Delivery Vehicles}

There are so many biocompatible, biodegradable cationic polymers which show the ability for targeted drug delivery. Polysaccharides are one of them which are isolated from natural sources and much better than synthetic polymers for making single and composite hydrogels. However, use of amphiphilic polysaccharides can be used for various biomedical applications mainly for preparation of various injectable polysaccharide structure-based hydrogels such as carboxymethyl chitin, hyaluronic acid, PTX-loaded modified hydrogels, Thermosensitive chitosan/dextran-polylactide/ glycerophosphate hydrogel, Gellan Gum ,Chitin-CaSO4-nanofibrin based injectable gel system, nanocomposite hydrogels, Chitosan, chondroitin Sulfate, photo-crosslinked gelatin methacrylate (GelMA) and thermosensitive chitosan/dextranpolylactide/glycerophosphate hydrogels and its functions in engineering cartilage tissue and injectable chemistries [21,22]. Other synthetic and semi-synthetic polymers such as Gelatin Methacryloyl; Chitosan Hyaluronic Acid; Dextran Gelatin; Gelatin Vinyl Acetate; Polycaprolactone; Polydimethyl Siloxane; polycaprolactone can be used to develop carrier systems. Besides polymers Vitamin E and C can be encapsulated within a carrier system in combination with drugs for delivering anti-cancer drugs at target sites. D-glucose is also used during nanoparticle formation to stabilize the gelatin/collagen-based matrix wall and reservoir wall for the deliveries of Calendula officinalis powder and oil. Plant proteins, essential oils and polysaccharides as biomaterials can be used to make safe carrier vehicles for delivering drugs. In addition, plant protein-based delivery vehicles can be used to deliver novel pharmaceutical products to patients. Protein based nano-systems can be used to deliver the active constituent at a sufficient concentration during the entire treatment period, directing it to the desired site of action without any side effect. Zein is a class of alcohol-soluble prolamine proteins found in maize endosperm is identified as safe excipient in combine herbal medicine with nanotechnology. Moreover, nano-structured systems might be able to potentiate the action of plant extracts, reducing the required dose and side effects, and improving activity. Nanoparticle based on the polysaccharide from Angelica sinensis acts as an efficient and safe non-viral gene vector [23]. Similarly, nanostructured lipid carriers (NLCs), composed of solid and liquid lipids, and surfactants are potentially good colloidal drug carriers. Virus delivery vectors are one among the many nano-materials that are being developed as drug delivery materials [24]. This chapter focuses on methods utilizing plant virus nano-particles (PVNs) synthesized from the Red clover necrotic mosaic virus (RCNMV). There are series of Ayurvedic herbs which are in use since ancient times but they are not scientifically authenticated for having much better formulations

\section{Authentic Drug Testing and Efficacy}

Ayurvedic Bhasmas were used as nanomedicine of herbometallic and mineral origin with particular attention to their structural aspects. Bhasmas have been used as nanomedicines which offer a huge potential for designing new drugs employing 
the concept of nanotechnology. Though it is very is a crucial issue to be addressed that all Ayurvedic formulations will need standardization of processing and formulations. Bhasmas are generally safe drugs for human beings in spite of the presence of seemingly toxic elements and compounds. Bhasmas are recommended for gaining the complete and reliable compositionprocessing-structure-effectiveness picture of these drugs. Nevertheless, more systematic nanomaterialistic investigations are needed on Bhasmas for their recommendation, gaining the complete and reliable composition-processing-structureeffectiveness picture of these drugs. Bhasmas are generally safe drugs for human beings in spite of the presence of seemingly toxic elements and compounds as indicated by recent studies using modern analytical techniques. The C. officinalis powder loaded nanoparticles significantly strengthened the anti-cancer effect towards human breast adenocarcinoma MCF7 cells and human hepatoma SKHep1 cells. Zinc oxide nanoparticles showed anti-diabetic activity of Jasada bhasma and the corresponding new drug, namely [25].

Ayurveda recommend Jasada bhasma (zinc based bhasma) as the treatment of choice for diabetes [26]. Modern medicine also recognizes the important role of zinc in glucose homeostasis. Yet, studies that validate the use of Jasada bhasma are few and un-comprehensive. There is an imminent need for a systematic study on physicochemical characterization, pharmacological efficacy, and toxicity assessment of several bhasma preparations to generate scientific evidence of their utility and safety. Interestingly, recent studies suggest that bhasmas comprise submicronic particles or nanoparticles. Thus; a bhasma-inspired new drug discovery approach could emerge in which several metal based nano-medicines could be developed. This would help in utilizing the age old, time-tested wisdom of Ayurveda in modern medicine. One such study on anti-diabetic activity of Jasada bhasma and the corresponding new drug, namely, zinc oxide nanoparticles, is briefly discussed, as an example.

\section{Development of Low Cost Vaccines}

For better health management of infants, juveniles and sub-adults low cost vaccines of few important diseases such as Hepatitis A and B, Tetanus, Diphtheria, Pertussis (DPT), Measles, Mumps, Rubella e from the WHO sources or from various states of the world. Most health insurance plans cover the cost of vaccines, but you may want to check with your insurance provider before going to the doctor. Under unified health policy by WHO health insurance plans can cover the cost of vaccines, and nations can do make subsidized the consultancy fee of doctor or insurance provider company should afford the fee before going to the doctor. It will need inclusion of affordable care act equally amended in the constitutional law of various states. USA has passed Affordable Care Act- the health insurance reform legislation in March 2010 that directs health plans to cover preventive services and eliminates cost sharing (such as co-pays and deductibles for certain services). Inaction of similar laws will increase the hope of people if they become eligible for preventive services including coverage of vaccines. Normally new plans can come to focus based on income, which will become mandatory and coverage options run side-by-side, after simple enrollment of various categories of children and adults also. Based upon household size and income private insurance plans can provide vaccines and life saving medicines at lower costs for which monthly premiums can be deducted from family father, group leader, community chief, ministry or from Government by its own.

There is a need of Children's Health Insurance Program (CHIP) on the basis of people and private partnership to cover JE, malaria, measles, mumps, DPT and hepatitis patients. Health insurance must cover series of vaccine shots, administration, and fee for child care and advice. School children can get full coverage of vaccine by making group insurance and private partners shared by school authorities, municipalities, boards and state governments. For management of payments in hospitals under long term insurance enrolled children at home and school can provide onetime credit card, for adults person finance cards, secured credit cards, travel credit cards and cash cards under essential vaccination provider services act. There must be a policy to ensure participation of schools in vaccination program and municipality boards to fill up necessary consent forms, and ensure vaccine availability, doctors, staffs and available methods of vaccine delivery cheap or free of cost. Many county health departments offer immunization services that have lower prices than private pharmacy service providers [27] .

\section{Adopting New Health Policy and Practices}

At global level there is a need to make essential policies that can support health and well being of the people [28]. There should be a new beginning by making and adopting all necessary changes in health policies, practices, drug formulation, scaling and their marketing. It should provide broad coverage on valuable tools, hospital appliances, diagnosis and medications/treatments on subsidized rates. There must be a common affordable care act that also looks after migrants, immigrants and nationals through one holistic approach. In such policies Federal Governments, Companies/stakeholders and people should place on a common platform for dialogue and communication for making mutualistic long term approach for health insurance coverage and investments. There must be common data depositories for sharing information, services, therapies, treatments and which will bind by only one law that allows only WFMA (World Forum for Medical Assistance) approved or cleared medical products for sale and purchase. It will also need adequate, well-controlled trials that could measure relevant clinical outcomes in the target population over an appropriate period. It will build confidence about therapeutic benefits outweigh its risks beyond the boundaries of religion, caste, creed and race. It will also reduce 
the difference between care provided in developed, developing and underdeveloped countries. In this direction new provisions have been made by UN health for all [28].

EU policies have revitalized the public health system and issued clear directives for improving and protecting human health, through modernization of medicine and therapeutics [29]. It is based on principles of proportionality and subsidiarity. EU established one Commission that has a role to support the EU Member States' efforts to protect and improve the health of their citizens and to ensure the accessibility, effectiveness and resilience of their health systems. As well as being a value in itself, health is a precondition for economic prosperity. For developing more sustainable health systems companies are allowed to invest in people's health, particularly through health promotion programs. Insurance coverage will reduce inequalities and tackling social exclusion.

Iranian health system is based on integration of medical education and health service provision, control of some important communicable and non-communicable disease [30]. There are noticeable health inequalities within the country; for instance, the difference between life expectancy between different provinces reaches 24 years. The same is through for almost all indicators excluding the vaccine coverage and access to primary health care which are above $90 \%$ nationwide. While the major burden of disease in the country as a whole and especially in the large metropolitans is a non communicable disease (NCDs) including cardiovascular disease (CVD), cancer and injuries. Iran is facing problems in its three south eastern provinces which have majority of falciparum malaria cases being foreigners who entered the country illegally.

Iran's health system has undergone several reforms in the past three decades with many challenges and successes. In year in 1983 important reform was establishment of the National Health Network which aimed to reduce inequities and expand coverage and access to health care in deprived areas.1 Later on several other reforms, such as the Family Physician Programme, integration of health services and medical education, the hospital autonomy policy, and the Health Sector Evolution Plan were also implemented by Iranian Government. The Health Sector Evolution Plan, launched in 2014, which directs Hospitaloriented transformation of the health service in all governmental hospitals to help address the substantial increase in health-care costs in the past decade.

Indian Government has launched National Health policy that will provide free medicines and "assured" health services to all and aims to reduce out of pocket health expenditure. It stresses on "preventive and promotive" healthcare and also has made a "target-oriented" commitment for elimination of diseases. The policy envisages the creation of National Health Care Standards
Organization which will formulate guidelines and protocols for healthcare while there is a provision of establishing a separate empowered tribunal for speedy resolution of disputes and complaints. Health minister said "In order to provide access and financial protection at secondary and tertiary care levels, NHP 2017 proposes free drugs, free diagnostics and free emergency care services in all public hospitals [31].

In addition there is need to frame new health policy for economically poor countries, and all insurance coverage should bear under UN by making an umbrella of nations to fight jointly against communicable and life style diseases. Before being implementation of such policies there should be one federal law to check illegal migration of people because they are becoming epicenters of communicable diseases and infecting people in developed and developing nations when come in contact. Travelling is also big challenge for travelers as they come under direct contact of residents infected with various pathogens and have infection by transmission vectors during their stay in hotels, site seeing and marketing.

\section{Alternative Medicines}

Alternative Medicine is a term used for overall forms of medicine except allopathy or conventional medicine. These are practiced for thousands of years as medicine, or as an alternate therapy. These are based on religion, sociality, culture and civilization and running date back hundred or even thousands of years. There are more than 100 different forms of alternative medicines still in practice all over the world other than western medicine. Most of these are native to different cultures and exist as traditional medicine, indigenous medicine or folk medicine etc. Alternative medicine consists of a wide variety of practices, products, and therapies-ranging from those that are biologically plausible but never tested or less tested for their harmful and toxic effects. Though, it is a tough job to properly diagnose alternative medicine, but proper testing and reforming methods and designing new formulations will change mindset of people and their scope will be increased. Therefore, by making slight changes drug composition and preparing a solid scientific explanation of any practice could make them part of science-based curricula in medical schools. Due to lack of analytical standards followed these are not used in any practice where treatment is based on scientific knowledge or proven experience. There is common opinion about alternative medicine these emphasize tradition, superstitions, belief in energies, pseudoscience, errors in reasoning, propaganda, fraud, or lies. Regulation and licensing of alternative medicine and health care providers varies between and within countries. There must be some new arrangement to follow common methods to prepare high action drugs, healing methods, mantras, asnas, healing methods and combine with available therapies and medicines jointly for good clinical successes . 
Every country, region or area has its own traditional system of health and medical cares such as for the Chinese it is acupuncture, for the French, magnetic healing; for the Germans, Heilpraxis; for the English, Herbalism; for India, Ayurveda with Siddha being widely practice in the southern part of the country; for Japan, Shiatsu etc. The most popular forms of alternative medicine are Ayurveda, Homeopathy, Naturopathy, Yoga, Acupuncture, Acupressure, Magneto therapy, Shiatsu, Herbalism, Meditation, Aromatherapy, Bach Flower Remedies, Chromo therapy, Diet therapy, Hydropathy and Reiki.

There is a myth that alternative medicine are disproven, unproven, or impossible to prove, and are excessively harmful in relation to their effect. There is common though in scientific community that these are therapies and have no diagnoses, hence, cannot become part of medicine or science-based healthcare systems. Alternative treatments are neither the same as experimental medicine, nor traditional medicine and have poor scientific methodology. World Congress of Alternative Medicines and the provisional program contained no less than 135 therapies. Perceived effects of alternative medicine may be caused by placebo; decreased effect of functional treatment. By making fine modifications, new additions and analytically appropriate we can potentially decrease their side effects. Lastly, if they add some potential and support modern medicine they should be credited as alternative therapies or composite medicine. Alternative medicine has grown in popularity and is used by a significant percentage of the population in many countries. There must be a suggestive view that alternative medicine be used together with functional medical treatments only then these can improve the targeted effect. On the basis of scientific methods and tools available these must be tested and make them more usable and remove all paradoxical views by adopting any alternative treatment in the category of medicine.

\section{Alternate Sources of Modern Medicines and Nutraceuticals}

Ocean is a largest habitat for different types of animals and plants mainly seaweeds which are rich source of nutraceuticals and bioactives which can be used as a basic source material for generating various types of pharmaceuticals [32]. Seaweeds are macroscopic, multicellular, marine algae which are rich source of minerals, iodine, calcium, protein, and oleic and alpha-linoleic acid acids. Seaweeds are used for food, medicine, fertilizer, filtration, and for industrial purposes. Most common plants are Laminaria (kelp), a brown algae and Ecklonia, green algae as sources of kunbu. Sargassum is a brown algae, and is source of haizao, Pyrphora is a red algae, as the source of zicai. Seaweeds are also rich in mineral elements and account for up to $36 \%$ of its dry mass. Important mineral macronutrients found in seaweeds are sodium, calcium, magnesium, potassium, chlorine, sulfur and phosphorus; the micronutrients include iodine, iron, zinc, copper, selenium, molybdenum, fluoride, manganese, boron, nickel and cobalt [33] .
Seaweeds are largest source of iodine large proportion of iodine. The highest iodine content is found in brown algae, with dry kelp ranging from 1500-8000ppm (parts per million) and dry rockweed (Fucus) from 500-1000ppm. Both red and green algae have somewhat lower contents of iodine that ranges from 100 $300 \mathrm{ppm}$ in dried seaweeds. Daily requirement of idoine $150 \mu \mathrm{g} /$ day, could be covered by using very small quantities of seaweed. Just one gram of dried brown algae provides from 500-8,000 $\mu \mathrm{g}$ of iodine and even the green and red algae. More-especially, purple nori used in Japanese cuisine provides 100-300 $\mu$ g in a single gram. In addition, seaweeds are one of the richest plant sources of calcium, and its content is typically about 4-7\% of dry matter [33]. Very simply one gram of dried seaweed provides 70 $\mathrm{mg}$ of calcium, compared to a daily dietary requirement of about $1,000 \mathrm{mg}$. This amount is higher than any non-milk based foods.

Seaweeds also contain higher percentage of protein content in seaweed varies somewhat. It is low in brown algae at $5-11 \%$ of dry matter, but comparable in quantitative terms to legumes at $30-40 \%$ of dry matter in some species of red algae [33]. Green algae contain protein content up to $20 \%$ of dry matter. Spirulina, a micro-alga, contains very high protein content, i.e., 70\% of dry matter. Seaweed contains several vitamins. Red and brown algae are rich in vitamins, mainly carotenes (provitamin A) and are used, in fact, as a source of natural mixed carotenes for dietary supplements. The content ranges from 20-170ppm. Red and brown algae contain vitamin $\mathrm{C}$ ranging from 500-3000ppm. Other vitamins are also present, including B12, which is not found in most land plants. Seaweed has very little fat, ranging from 1-5\% of dry matter, although seaweed lipids have a higher proportion of essential fatty acids than land plants. Green algae, whose fatty acid make-up is the closest to higher plants, have a much higher oleic and alpha-linoleic acid content. Red algae have a high EPA content, a substance mostly found in animals, especially fish. Seaweed also contains higher fiber content that ranges from $32 \%$ to $50 \%$ of dry matter. The percentage of soluble fiber is different for different algae, it accounts $51-56 \%$ of total fibers in green (ulvans) and red algae (agars, carrageenans and xylans) and for $67-87 \%$ in brown algae (laminaria, fucus, and others) [33]. Soluble fibers are generally associated with having cholesterol-lowering and hypoglycemic effects

Porphyra is well known seaweed that is largely used for food. It is red to purple color algae that is used as vegetable and known as zicai. In Japan it is known as nori, the red pigments are lost and the final product has a dark greenish color. Nori is used to wrap sushi and for making numerous snacks. Kunbu is highly nutritious and other common food item that has low cost and prevalent in China. In Japan it is known as Kombu that is usually sold in 5-6-inch dried pieces and can be found in health food stores and Japanese groceries. It is also sold as kombu that cooks quickly, vinegared, shaved kombu that needs little or no cooking, boiled, soy sauce flavored kombu and lightly lightly pickled. The powdered kombu is sprinkled on food or used in drinks. A third 
seaweed widely used in Japan is Undaria pinnatifida which is known as wakame [34].

Haizao and Kunbu used for medicinal purposes. These are salty and cold, and enter the liver, lung, and kidney meridians. Both can clear heat, transform phlegm, soften hardness, and dissipate nodules. They can also promote urination and reduce edema. In clinical practice, they are often used together to treat nodules such as goiter and scrofula. Haizao is stronger in transforming phlegm and dissipating nodules, and it is more suitable for treating goiter and scrofula. Kunbu is stronger in softening hardness and reducing congealed blood; it is more suitable for treating liver-spleen enlargement, liver cirrhosis, and tumors. One of the best known formulas with the seaweeds is Haizao Yuhu Tang, or the Sargassum Decoction for the Jade Flask. This formula contains 12 ingredients includes Sargassum, Ecklonia, and Laminaria. It is used to treat a goiter. From ocean so many seaweeds have been adopted into formulas for treating other soft swellings, including ovarian cysts, breast lumps, lymph node swellings, lipomas, and fat accumulation from simple obesity [34]. There are important marine plants Caulerpa, Fucus, Gracillaria, Laminaria, Macrosystis, Monostroma, porphyra which are used for medicinal and nutraceutical purposes.

\section{Plant Toxins}

Many constituents of plants elicit pharmacological or toxicological effects in man and animals. Plant toxins are toxic secondary plant metabolites which naturally occur in food, feed, weeds and ornamental plants. These are cidal in nature and show anti-therapeutic effects against bacteria, viruses, protozoans, animals, and microorganisms. The chemical diversity phycotoxins relates to saponins, mimosine, vsicotoxins, linamarin, solasodamine, amygdalin and epipodophyllotoxins which are used for different therapeutic purposes. There are plant species which produce compounds that are only dangerous to people and develop allergic reaction upon exposure. Ricinus communis seeds contain ricin, an extremely toxic and watersoluble ribosome-inactivating protein. Also present are ricinine, an alkaloid, and irritant oil. Drynaria, commonly known as basket ferns, is a genus of ferns in the family Polypodiaceae. It is epiphytic in nature economically important medicinal plant. Achyranthes aspera Linn. (Amaranthaceae) is also widely used for medicinal purposes.

Berry plants are poisonous; its consumption shows sedative effect on cardiac muscle tissue and can cause arrest. Seeds and roots contain cardiogenic toxins which cause both severe gastroenteritis and heart palpitations if consumed. Tropane alkaloids, scopolamine, hyoscymine and atropine are also important plant poisons, especially found in seeds and flowers. Young plants and seeds are poisonous, causing nausea, muscle twitches, paralysis, and often death. African plant contain ouabain a potent cardiac glycoside that, when sufficiently concentrated, can induce cardiac arrest by binding to and inhibiting the action of the sodium-potassium pump. Terrestrial weeds also provide low cost, more potential, weed Guduchi (Tinospora cordifolia) is used for production of many medicines.

\section{Future Directions}

Regardless of the availability of therapeutic options, other than allopathy the overall curative potential of $90 \%$ of existing drugs is not very high. Either they provide mild cure without side effects. Contrary to this allopathy makes use of strong medicines to cure, but impose severe side effects. Though, homeopathic medicines are not only sweet, low cost and easy to take. Homeopathic remedies which are prescribed in low doses can be potentiated for much better action after making slight changes. It will generate intermittent biological signals and stimulate the organism's allostatic biological stress response network at much faster rate, evoke nonlinear modulatory, selforganizing change. After rigorous authentication homeopathic remedies can be proposed as source of nanoparticles for timedependent sensitization via non-pharmacological effects on specific biological adaptive and amplification mechanisms. Normally, these homeopathic remedies work effectively without any side effect but at a slow pace that can be enhanced. By making little modifications based on nanomedicine distribution and targeting besides acute diseases/disorders these could be used to treat chronic diseases like asthma, heart problems, cancer etc. The danger of allopathic medicines is that they do not cure but suppress.

Further, by adopting molecular analytical methods, nanoparticle nature of remedies can be distinguished easily from conventional bulk drugs in structure, morphology, and functional properties. Further, in biological testing systems therapeutic outcomes of drug can be reinvestigated for its ability respond with diverse groups of micro-organisms or a single one. After exploring ligand and receptor interactions very closely structure, function, and applications of plant natural products such as proteins, peptides, lipids, vitamins, carbohydrates, complexes, essential oils, and enzymes can be partially modified chemically to make them more usable and active. Further, in preformed cellular systems nano-drugs can be tested for its novelty. However, synthetic allopathic drugs generate stress or heterotypic biological threat, cumulative, cross-adapted biological maladaptations and even much severe morbidities. Therefore, after identification of biological activity in different cell and animal systems a nano-size drug formulation can provide large therapeutic coverage instead of conventional drug formulae. It can be achieved by making more recent advancements in the fields of nanotechnology and biomedical sciences.

Further, old thoughts prevalent in society regarding use of alternative medicine can be improved by making authentication of drug molecules by identifying their nature. It will increase their scientific validity. There are paradoxical views about 
its principle, formulation and action. Old mindsets say that alternative medicine has no scientific basis and is a waste of scarce research resources. Now time has reached that among all prevalent alternative treatments some of them are classical in nature and are adoptable. A positive view can develop by employing molecular and cellular level authentication and testing of these medicines could provide some substantial results that are more useful because of region specific availability of drug formulation/s. Through investigations are needed to widely screen nature of bio-molecules and testing their purity. However, all different medicines from different systems can be screened through a more authentic analytical system/s that can correctly rule out theory-driven research and can emphasize therapeutic use of nano-materials in living systems, mechanisms actions and translational uses in nano-medicine. Therefore, all nano-size materials from Ayurvedic, homeopathic, Unianai and Siddha formulations must tested for their physiological action. Because all physiologically active substances pass through cell membranes, the capillary wall and show their action on the function of cells and organs after biological processing. All nanomedicines developed from plant biomaterials interact directly with functioning organs, cells, subcellular structures, calcium channels, sodium-potassium pump. Hence, there is a need to develop composite system of medicine by using nanotechnologybased drug delivery systems and herbal medicines that can potentially work against majority of the bacterial strains and fungal colonies. Nanosystems can deliver the active constituent at a sufficient concentration during the entire treatment period, directing it to the desired site of action. Conventional treatments do not meet these requirements. However, for shifting from old medicinal system to new medicinal system there is utmost need to design more value-added therapeutic products that may show far greater effectiveness as a drug. There must be fusion of traditional, alternative, biomedical and nano-medicine for acceptability of drug designs and their use at international level. There is a need to find alternatives of vaccines because both attenuation and inactivation may fail in future there will remain possibility of reversal of epidemics at larger scale. Both viral and bacterial strains become resistant to most of the conventional drug, if in future vaccines also come under resistant or change in antigenic behavior than it will be very hard to any medicinal system to stop larger epidemics.

Due to failure of so many drug structures and rising number of patients there is an increasing trend of global consumption of herbal products and medicines (Figure $5 \& 6$ ). It is right time to enhance drug quality, effectiveness, and coverage in different seasons. From clinical point of view there is need to include them in in pharmacovigilance systems. There is utmost need to identify the risks associated with the use of herbal medicines. Because of drug induced side effects, it becomes an issue of great public health importance [35]. More specifically for use of these drugs thorough toxicity assessment alongside active pharmacovigilance are highly needful for safe use and protect public health. The development as well as implementation of the regulation of traditional or herbal medicines in different parts of the world is often confronted with several challenges (Figure 5 \& 6). Challenges often encountered and common to many countries are those related to regulatory status, assessment of safety and efficacy, quality control, safety monitoring and inadequate or poor knowledge about traditional, complementary/alternative, and herbal medicines within national drug regulatory authorities. There is an important challenge related to the regulatory status of herbal medicines. a herbal medicinal product or "dietary ingredient" is toxic or not safe for use. Additional major challenge in many countries is the fact that regulatory information on herbal medicines is often not shared between regulatory authorities and safety monitoring or pharmacovigilance centers [35].

There are challenges related to assessment of safety and efficacy. There is no gainsaying the fact that the requirements as well as the research protocols, standards and methods needed for the evaluation of the safety and efficacy of herbal medicines are much more complex than those required for conventional or orthodox pharmaceuticals [35]. A single herbal medicine or medicinal plant may contain hundreds of natural constituents, and a mixed herbal medicinal product may contain several times that number. Suppose every active ingredient were to be isolated from individual herb from which the herbal medicine is formulated or produced, the time and resources required would be tremendous. Such an analysis may practically be impossible especially where an herbal product is a mixture of two or more herbs [36]. There are major challenges regarding quality control of herbal medicines. The quality of the source materials used in the production of herbal medicines must be determined for safety and efficacy of herbal remedies. Generally, the quality of source materials is dependent not only on intrinsic (genetic) factors, but also on extrinsic factors like environmental conditions, good agricultural, and good collection practices (GACP) for medicinal plants, including plant selection and cultivation. It is the combination of these factors that makes it difficult to perform quality controls on the raw materials of herbal medicines [37,38]. According to good manufacturing practice (GMP), correct identification of species of medicinal plants, special storage, and special sanitation and cleaning methods for various materials are important requirements for quality control of starting materials.

There are challenges related to safety monitoring of herbal medicines. Major challenges are related to quality control of finished herbal medicinal products, especially mixture herbal products. It is very difficult to test ingredients included from all the plants or starting materials [36,37]. Thus, the general requirements and methods for quality control of finished herbal products remain far more complex than for other pharmaceuticals. To ensure safety and efficacy of herbal 
medicines, there must quality assurance and control measures such as national quality specification and standards for herbal materials by making important trials in sophisticated laboratories [39]. There must be authoritative control on herbal medicines, for proper labeling, and licensing of schemes for manufacturing, import and marketing of herbal medicines.

\section{Conclusion and Recommendations}

So many existing drugs belong to modern system of medicine become outdated and they are failed fight against drug resistant strains. It has resulted into global acceptance and use of herbal medicines and related products on large scale. Besides, available drug formulae there are more than $30 \%$ of drugs which have therapeutic efficacy below $90 \%$. Most of the existing drugs are costly and toxic in nature and generate many side effects. All such drugs must be replaced completely by most recent drug structures. However, to have new bio-actives and natural drug formulations plant biomaterials such as extracts, pure compounds, powders, latex, essential oils and their components could be used to develop new composite medicines which will be cost effective, competitive and do not need any carrier system for delivery. There are thousands of bio-organics available from plants which have diverse pharmacologic and clinical effects. These plant origin bio-organic compounds can be used to prepare nano medicines, its metallic conjugates, peptide and protein complexes that should be biocompatible and able to potentiate the action inside the cells and organs. No doubt drug structures and effectiveness can be optimized by combining herbal medicine with nanotechnology. These nanostructures might be able to potentiate the action at cellular level, reducing the required dose and side effects, and improving activity. For fighting clinical pathogens selective PARP cleaved strongly rationalized superior apoptotic inducers are available in plant cells. The only need is to isolate and identify their behaviors in different cell systems for extensive clinical use. For making CVD drugs the easiest way is PEGylation that plays important role in drug delivery, mainly its distribution in myocardial cells. More specifically mono-PEGylated conjugates of Radix Ophiopogonis polysaccharide (ROP) found clinically relevant in animal models for treatment of myocardial infarction ischemia/reperfusion (IR) model. Besides, nano-pharmaceuticalsm magnetic nanomedicine based on diosgen in showed anti proliferative, migration inhibiting and apoptosis inducing properties against breast cancer.

Besides developing new drugs, there is a need to analyze use and dosage effects of so many traditional and ethno medicines. In addition, all issues relating to adverse reactions have been resolved by using authenticated analytical methods for drug testing. It will also need regulatory policies on herbal medicines for standardization of drug formula to strengthen the therapeutic index of herbal medicines on a global scale. Relevant regulatory authorities in different countries of the world need to be proactive and continue to put in place appropriate measures to protect public health by ensuring that all herbal medicines approved for sale are safe and of suitable quality. For increasing knowledge all medicine providers such as physicians, nurses, and pharmacists supporting staff should know the basic diagnosis of disease, prescription and its duration. They should bear huge responsibility of safety monitoring of medicinal products and their proper use according to need of the patient. It is essential to create an atmosphere of trust to facilitate adequate sharing of knowledge about the use and safety of herbal medicines. In fact, the education of healthcare professionals, providers of herbal medicines, and patients/consumers is vital for the prevention of potentially serious risks from misuse of herbal medicines.

There are thousands of active principles form different plant species which are not tested for their multiple biological activities or either they are the phase of regular trials. There are millions of medicinal plants products/extracts that can be used as potential products for production of highly innovative phyto pharmaceuticals. Most of them are known to possess antibacterial properties against gram-positive bacteria, others to inhibit bacterial colonization by blocking specific carbohydrate receptors involved in host-bacteria interaction. Their mode of action can be used to design alternative anti-adhesion therapy. There are series of biomaterials which are new to the market, still unidentifiable or remain unscreened till date but may be of high therapeutic use. It will certainly give new insights in developing potentially new highly effective drug formulations not only to cure simple diseases but also use as life saving drugs after having proper composition, testing, processing and marketing. Further to strengthen overall health system there is need to combine individual interventions and public health needs not only economic grounds but on the basis of humanity to make the system cost-effective through social, political, clinical and industrial initiatives. Before start using drug prescriptions clinicians should possess appropriate knowledge for proper diagnosis and treatment decision-making capacity. Furthermore, individual healthcare provider should also show sufficient commitment toward understanding the use of herbal medicines. Finally, as with other medicines for human use, it has become mandatory that herbal medicines are covered in every country of the world by a drug regulatory framework to ensure that they conform to required standards of safety, quality, and efficacy. By increasing awareness about early diagnosis and right medicine a sustainable health-care system can be established by keeping aside economic imbalances at global level with the aid of WHO. Further, combination of various therapies such as Ayurvedic massages, herbal therapy, yoga and traditional surgery has imposed magnetic effect and attracting more patients towards these therapies. However, due to extended potential of traditional and ethnomedicine drug market booming because of more reliability and promise shown by natural medication. This accounts for a huge potential in the up and coming times for herbal medicines (Figure $5 \& 6$ ). 


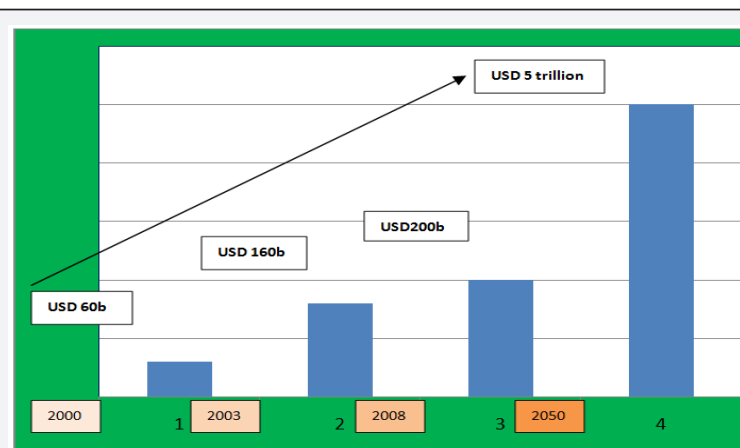

Figure 5: Showing global market value of herbal industry is increasing at $7 \%$ annual growth.

WHO member states with traditional medlcine pollcy

WHO member states regulating herbal medicine

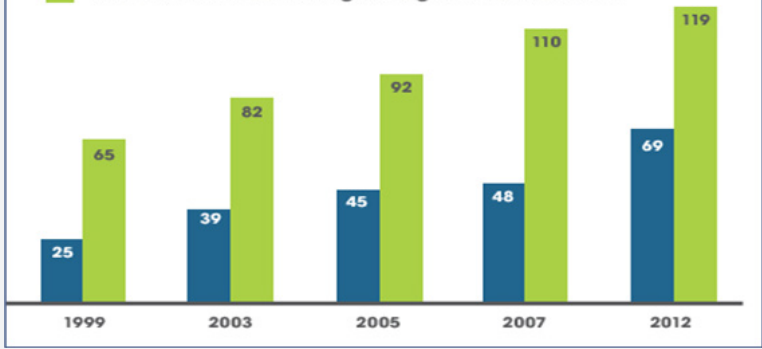

Figure 6: Showing worldwide regulation of traditional medicine.

\section{References}

1. Martins E (1996) The growing use of herbal medicines: issues relating to adverse reactions and challenges in monitoring safety. Front Pharmacol 4: 177.

2. Raynor DK, Dickinson R, Knapp P, Long AF, Nicolson DJ (2011) Buyer beware? Does the information provided with herbal products available over the counter enable safe use? BMC Med 9: 94.

3. Acharya D, Anshu S (2008) Indigenous Herbal Medicines: Tribal Formulations and Traditional Herbal Practices. Aavishkar Publishers, India.

4. Laguerre MS (1987) Afro-Caribbean folk medicine. Bergin \& Garvey, USA.

5. Gilani AH (2005) Role of Medicinal Plants in Modern Medicine. Malaysian Journal of Science 24(1): 1-5

6. De Sa C (1993) The preventive-curative conflict in primary health care. Health Millions 1(2):14-17.

7. Bernard SB, Aurelia R, Sandrine D, Egon J (2000) Evaluation of randomized controlled trials on complementary and alternative medicine. International Int J Technol Assess Health Care 16 (1): 13-21.

8. (2016) National Policy on Traditional Medicine and Regulation of Herbal Medicines-Report of a WHO Global Survey, Geneva, Swizerland.

9. Heinrich M, Pieroni A, Bremner P (2005) Plants as medicines. In: Prance G, Nesbitt M (Eds.), The Cultural history of plants. Routledge, UK, pp. 205-238.

10. Global action plan for the prevention and control of NCDs 2013-2020.

11. (2014) WHO Global status report on non-communicable diseases. 298

12. Hiramatsu K, Katayama Y, Matsuo M, Sasaki T, Morimoto Y, et al. (2014) Multidrug resistant Staphylococcus aureus and future chemotherapy. J Infect Chemother 20(10): 593-601.
13. Peter BB (2001) Drug resistance in malaria.

14. White NJ (2004) Antimalarial drug resistance. J Clin Invest 113(8): 1084-1092.

15. Aginam 0 (2002) From the Core to the Peripheries: Multilateral Governance of Malaria in a Multi-Cultural World. Chic J Int Law 3(1): 1-19.

16. Marsh K (1998) Malaria disaster in Africa. Lancet 352(9132): 924.

17. Richman DD, Morton SC, Wrin T, Hellmann N, Berry S, et al. (2004) The prevalence of antiretroviral drug resistance in the United States. AIDS 18: $1393-1401$.

18. Alistair DC, Alecia AF, Murray (2010) Emergence of Increased Resistance and Extensively Drug-Resistant Tuberculosis Despite Treatment Adherence, South Africa. Emerg Infect Dis 16(2): 264-271.

19. Ni X, Suhail MM, Yang Q, Cao A, Fung KM, et al. (2012) Frankincense essential oil prepared from hydrodistillation of Boswellia sacra gum resins induces human pancreatic cancer cell death in cultures and in a xenograft murine model. BMC Complement Alter Med 12: 253.

20. Saikia C, Gogoi P, Maji TK (2015) Chitosan: A Promising Biopolymer in Drug Delivery Applications. J Mol Genet Med S4-S6.

21. Shukla R (2001) Munir Cheryan Zein: the industrial protein from corn Industrial Crops and Products 13: 171-192.

22. Deng W, Fu M, Cao Y, Cao X, Wang M, et al. (2013) Angelica sinensis polysaccharide nanoparticles as novel non-viral carriers for gene delivery to mesenchymal stem cells. Nanomedicine 9(8): 1181-1191.

23. Fang CL, Saleh SA, Fang JY (2013) Nanostructured Lipid Carriers (NLCs) for Drug Delivery and targeting. Recent Pat Nanotechnol 7(1): 41-55.

24. Lam PL, Kok SH, Bian ZX, Lam KH, Tang JC, et al. (2014) D-Glucose as a modifying agent in gelatin/collagen matrix and reservoir nanoparticles for Calendula officinalis delivery. Colloids Surf B Biointerfaces 117:277-283

25. Rinku D, Umrani, Kishore MP (2015) Jasada bhasma, a Zinc-Based Ayurvedic Preparation: Contemporary Evidence of Antidiabetic Activity Inspires Development of a Nanomedicine 2015 (2015): 9.

26. (2017) Low-cost vaccine manufacturing by MIT.

27. World Health Organization (2013) Zsuzsanna Jakab WHO Regional Director for Europe; Towards people-centred health systems: An innovative approach for better health outcomes, pp. 1-34.

28. Jan H (2011) School and Health 21, 2011, Health Literacy through Education Developing new European Health Policy and Health Systems-Health 2020.

29. Bahram H, Hassan J (2016) Iran's health care system in transition. Lancet 387(10013): 29-30.

30. Nadda JP (2017) Cabinet approves National Health Policy 2017; A huge milestone in the history of public health in India.

31. Round FE (1962) The Biology of the Algae. Edward Arnold Ltd.

32. Christian W, Kai B (2012) Seaweed Biology: Novel Insights into Ecophysiology, Ecology \& Utilization, Springer-Verlag Berlin Heidelberg, Germany.

33. Matsuzaki S, Iwamura K (1981) Application of Seaweeds to Human Nutrition and Medicine. Nahrung aus dem Meer/Food from the Sea 13: 162-185.

34. Zhou J, Ouedraogo M, Qu F, Duez P (2013) Potential genotoxicity of traditional Chinese medicinal plants and phytochemicals: an overview. Phytother Res 27(12): 1745-1755. 
35. WHO (2004) WHO Guidelines on Safety Monitoring of Herbal Medicines in Pharmacovigilance Systems. Geneva, Switzerland.

36. WHO (2005) WHO global atlas of traditional, complementary and alternative medicine. In: Ong CK, Bodeker G, Grundy C, Burford G, Shein (Eds.), Geneva, Switzerland.

37. WHO (2005) National Policy on Traditional Medicine and Regulation of Herbal Medicines. Report of a World Health Organization Global Survey. Geneva, Switzerland.
38. Roberts JE, Tyler VE (1997) Tyler's Herbs of Choice. The Therapeutic Use of Phytomedicinals, The Haworth Press, USA

39. Rodrigues E, Barnes J (2013) Pharmacovigilance of herbal medicines: the potential contributions of ethnobotanical and ethnopharmacological studies. Drug Saf 36(1): 1-12.

\section{Your next submission with Juniper Publishers} will reach you the below assets

- Quality Editorial service

- Swift Peer Review

- Reprints availability

- E-prints Service

- Manuscript Podcast for convenient understanding

- Global attainment for your research

- Manuscript accessibility in different formats

(Pdf, E-pub, Full Text, Audio)

- Unceasing customer service

Track the below URL for one-step submission https://juniperpublishers.com/online-submission.php 